\title{
Estrategias de marketing territorial en una región transfronteriza: Tamaulipas-Texas
}

\section{Strategies of place marketing in a cross-border region: Tamaulipas-Texas}

\author{
Xavier Oliveras González* \\ El Colegio de la Frontera Norte, Departamento de Estudios \\ Urbanos y Medio Ambiente, Tijuana, México
}

Recibido: 18 marzo de 2015. Aprobado: 2 de julio de 2015.

\begin{abstract}
Resumen
En un contexto de competencia espacial a nivel global y de reivindicación de las escalas local y regional, los lugares han adoptado el marketing territorial como estrategia de desarrollo territorial. Para estas estrategias se movilizan actores y recursos con el objetivo de captar inversiones, turismo y residentes. En las regiones fronterizas se promueve dicha localización y las interrelaciones transfronterizas, a la vez que se construye una marca territorial transfronteriza. Se analiza el caso de la frontera Tamaulipas, México-Texas, Estados Unidos donde, desde fines del siglo XIX, se han desarrollado tres líneas estratégicas principales: para la captación de residentes e inversores agrícolas; de turistas; y de inversores industriales. Aunque en algunas ocasiones las estrategias son producto de la colaboración transfronteriza, mayoritariamente éstas han sido desarrolladas por los actores territoriales del sur de Texas; asimismo, se basan en las asimetrías territoriales entre ambos lados de la frontera. Ambas dinámicas refuerzan la diferenciación fronteriza en lugar de la unidad transfronteriza. La investigación sigue una metodología cualitativa, habiendo realizado un análisis de contenidos a partir de la documentación de las campañas de marketing territorial.
\end{abstract}

Palabras claves: marketing territorial, marca territorial, región transfronteriza.

* Doctor en Geografía. Investigador y coordinador, profesor de la Especialidad en Estudios de la Frontera MéxicoEstados Unidos. 


\begin{abstract}
In a context of global spatial competence and of local and regional scales resurgence, places have adopted place marketing as strategy of regional development. For those strategies are mobilized territorial agents and resources in order to attract investments, tourism and residents. Border regions promote the border localization and cross-border interrelations, and construct cross-border place brands. It is analyzed the case of Tamaulipas, Mexico-Texas, USA border. Since the end of XIX century three main strategies have been developed: to attract residents and agriculture investors; tourists; and industrial investors. Although in some occasions strategies are the result of cross-border collaboration, most strategies have been developed by South Texas territorial agents. Furthermore, strategies are based on spatial asymmetries between both sides of the border. Both dynamics reinforce border differentiation instead of cross-border unity. The research follows a qualitative methodology: it has been conducted a content analysis based on the documentation of place marketing campaigns.
\end{abstract}

Keywords: place marketing, place brand, cross-border region.

\title{
Introducción
}

En un contexto de competencia espacial a nivel global y de reivindicación de las escalas local y regional, se ha difundido ampliamente la aplicación de estrategias de marketing territorial (place marketing) por parte de los lugares (ciudades, regiones, etc.) para atraer y retener residentes, inversionistas, visitantes, trabajadores o estudiantes. Aunque dichas estrategias se vienen dando desde mediados del siglo XIX, éstas han sido más habituales a medida que la elección de un lugar pasa a basarse en los llamados soft factors, como la imagen territorial. En el presente artículo se presta atención al marketing territorial desarrollado en y desde las regiones transfronterizas, lugares particulares escasamente analizados en las investigaciones sobre este fenómeno. Como se detallará más adelante, los estudios que tratan estas regiones se focalizan mayoritariamente en experiencias europeas, cuyo análisis se apoya en dos supuestos: el manejo mediante procesos de cooperación transfronteriza, ampliamente extendida en Europa; y las semejanzas a ambos lados de la frontera (culturales, socioeconómicas, usos del suelo, etc.), lo que facilitaría la construcción de una marca de unidad transfronteriza. A nivel mundial estos supuestos no se cumplen siempre, siendo el contexto europeo una excepción más que 
la norma. Por ello, en esta investigación se ahonda en un contexto fronterizo distinto, pero más común, donde la práctica de la cooperación es escasa y las asimetrías entre ambos lados son notables. Se toma el caso de la región transfronteriza Tamaulipas-Texas (México-Estados Unidos).

En la frontera México-Estados Unidos las estrategias de marketing territorial se iniciaron en el último tercio del siglo XIX, desarrolladas en gran medida por actores de las regiones fronterizas de Estados Unidos -eminentemente la élite angloamericana-, los cuales han hecho uso de la frontera y el "otro lado" como recurso para la promoción de "su lado". Culver (2009) y St. John (2009) lo muestran para el sur de California, donde las estrategias adquieren un carácter transfronterizo al promocionar también Baja California, México. Como se mostrará, en el sur de Texas se ha procedido de manera similar en relación a Tamaulipas. A pesar de las similitudes entre las estrategias de las regiones fronterizas de California y Texas, existe una diferencia sustancial en lo que respecta a la construcción y uso de lo "mexicano", debido al diferente contexto fronterizo -en relación al concepto en inglés frontier-, de avance de la "civilización", de conquista y colonización angloamericana sobre la naturaleza y las poblaciones indígena y mexicana. Cabe recordar que el sureste de Estados Unidos era parte de México hasta 1848, cuando se formalizó su conquista por el primero. En California aquel proceso culminó hacia 1870, por lo que las estrategias se elaboraron en un contexto post-frontier, es decir, de reinterpretación del mismo. Por el contrario, en el sur de Texas las primeras estrategias, a partir de 1880, se pensaron paralelamente a dicho proceso, a la vez que formaban parte de aquél.

En el caso de estudio, el desarrollo de las estrategias con dimensión transfronteriza se da principalmente desde el sur de Texas, y se basan en una asimétrica representación espacial de los lados texano y tamaulipeco / mexicano. Tanto la captación como la distribución espacial a cada lado de la frontera de las inversiones, actividades económicas, residentes y turistas responden y perpetúan la misma diferenciada representación; igualmente se da en la distribución de funciones en caso de colaboración transfronteriza. Asimismo, la evolución de las estrategias desde fines del siglo XIX hasta la actualidad responde a la ocupación, transformación y construcción de aquel territorio.

Los resultados presentados son fruto de la primera fase de la investigación. Se ha procedido a 1) identificar las estrategias, 2) sus objetivos, 3) los actores territoriales, 4) las acciones y/o los medios para la promoción, 5) las poblaciones y/o sectores target a las que van dirigidas y 6 ) las marcas territoriales construidas (y los elementos que las componen). El arco temporal de estudio comprende desde la delimitación de la actual frontera entre México y Estados Unidos hasta la actualidad (exceptuando el reciente y 
aún emergente desarrollo del sector energético derivado de la Reforma Energética de México). Para ello se ha revisado la bibliografía existente sobre desarrollo regional del área de estudio y la documentación (publicaciones, publicidad y páginas web) de las campañas de marketing territorial. Asimismo se ha vaciado, de acuerdo a una búsqueda de conceptos clave, un conjunto de publicaciones periódicas (digitales y digitalizadas): revistas regionales generalistas (Texas Monthly y Somos Tamaulipas), revistas geográficas (The National Geographic Magazine), revistas especializadas en marketing industrial y turístico, generales (Site Selection Magazine) y regionales (Texas \& Turismo, De Shopping en el Valle de Texas y Mexico Industry Tamaulipas), y periódicos locales (The Brownsville Herald, The Monitor, Corpus Christi Caller-Times y El Mañana). Se ha conducido un análisis de contenidos de la información recabada de acuerdo a las variables analíticas señaladas.

\section{Marketing territorial y regiones transfronterizas}

El marketing territorial (place marketing) se suele conceptualizar de forma sencilla como un proceso social por el cual un país, una región, una ciudad o una destinación turística, entre otros lugares, compite por la atracción de recursos económicos y humanos. En este proceso el lugar es, metafóricamente, sujeto y objeto; obviamente, los sujetos no son los lugares per se, sino algunos actores territoriales en representación del lugar, que los subjetivan. El marketing territorial se compone de dos procesos distintos, aunque interrelacionados: la creación, difusión y mantenimiento de una marca territorial (place branding) y la promoción territorial (place promotion) (Witte, 2014; Andersson, 2010). Por un lado, la marca territorial es una entidad construida con los aspectos sobre el lugar (geográficos, históricos, culturales, económicos, de identidad, etc.) positivos, únicos y distinguibles. Aunque se trate de conceptos parecidos, la marca se distingue de la imagen territorial por cuanto que ésta última incluye también los aspectos que son negativos y menos deseables de ser asociados con el lugar objeto de marketing. La promoción, por otro lado, se refiere a la comunicación del lugar y/o la marca, realizada por medio de distintos canales o acciones (publicidad, organización y participación en eventos, publicación de textos y material gráfico, etc.).

El marketing territorial tiene tres funciones básicas (Andersson, 2010): a) presentar información del lugar y sobre el lugar, b) incrementar la demanda en ese lugar y c) diferenciar un lugar de otros. Éstas se dirigen a la captación y/o mantenimiento de targets específicos (inversionistas, consumidores, turistas, residentes, empleados y estudiantes), 
por lo que en concreto se refiere a localizaciones geográficas específicas: viviendas y áreas residenciales; áreas para el desarrollo de actividades económicas (suelo agrícola, áreas de oficinas, áreas industriales, centros comerciales, etc.); áreas para el desarrollo comunitario y recreativo (parques, espacios naturales, equipamientos culturales y deportivos, centros educativos, bares y restaurantes, playas, etc.); complejos turísticos; y suelo para inversión en bienes raíces. Aunque se trate de lugares específicos, éstos no son promocionados de forma aislada sino que en las estrategias de marketing territorial se relacionan con "una" totalidad del territorio (de la que están excluidos los aspectos y lugares negativos e indeseables). En tanto que herramienta de desarrollo territorial, el marketing se asocia sobre todo con fines económicos, aunque no por ello están excluidos los sociales y culturales (recuperación y refuerzo de la identidad local y regional, valorización del patrimonio cultural y ambiental, entre otros). Asimismo, las estrategias de marketing se apoyan en la trasladación de los intereses particulares de los actores que lo desarrollan con un interés general del territorio, por lo que a nivel analítico no se deben confundir.

Como se apuntaba en la introducción, el grueso del estudio sobre marketing territorial se focaliza en lugares "individuales"; por el contrario, se ha prestado escasa atención a los espacios interregionales (regiones transfronterizas, redes de ciudades y de regiones, y macrorregiones). En el marketing interregional dos o más lugares, con o sin contigüidad geográfica, colaboran para el desarrollo de una estrategia común. La escasa atención recibida se debe a que, epistemológicamente, la interregionalidad entra a priori en contradicción con las funciones del marketing territorial (Oliveira, 2014; Pasquinelli, 2013). La premisa conceptual del marketing es la competencia entre lugares, mientras que para la estrategia interregional lo es la cooperación entre lugares. Sin embargo, competencia y cooperación no son mutuamente excluyentes: en el marco de una competencia espacial global la cooperación entre lugares tiene por objeto mejorar la competitividad frente otros lugares, lo que Pasquinelli (2013) define como "coopetición".

Superada aquella aparente contradicción, solo recientemente se ha empezado a analizar el marketing interregional, incluido el transfronterizo (Hospers, 2004; 2006; Ioannides, Nielsen y Billing, 2006; Andersson, 2007; 2010; Prokkola, 2007; Nilsson, Eskilsson y Ek, 2010; Šerić y Vitner, 2011; entre otros, hasta la celebración de la primera Conference on Interregional Place Branding en 2014 en Lübeck, Alemania). Las regiones transfronterizas ofrecen un caso particular, con retos conceptuales propios, derivados de la contigüidad geográfica y la vecindad entre ambos lados de una frontera internacional. Su análisis implica la necesidad de dar respuesta a varias preguntas: ¿las marcas territoriales están sujetas a las fronteras entre Estados-nación? ¿̨la frontera y el otro lado pueden constituir un elemento de la promoción y marca territoriales? ¿cómo evitar y/o superar 
los efectos de la división política en los procesos de marketing territorial? ¿estas estrategias niegan o cuestionan la existencia de las fronteras entre Estados-nación? y ¿cómo la frontera interviene en la relación entre los actores territoriales?

El corpus teórico se ha construido sobre el estudio de los casos europeos, en tanto que en Europa el marketing transfronterizo es un fenómeno ampliamente extendido, con una creciente popularidad y una gran diversidad de escalas (desde la local a la macroregional) y alcances (comercio, turismo, industria, etc.) (Futmair, 2014; Lamour, 2014; Witte, 2014). Las regiones transfronterizas de Escandinavia han recibido una atención especial, destacando el análisis de la Euroregión Öresund, en la frontera Dinamarca-Suecia (Andersson, 2010; 2007; Hospers, 2006; 2004). Aquella experiencia se ha tomado como un ejemplo de "buenas prácticas" y utilizado como punto de referencia para otros estudios. En esta dirección, para América del Norte, donde hay una menor experiencia, se han realizado también algunas investigaciones, donde el caso de Cascadia (en la frontera Estados Unidos-Canadá) es comparado con aquél (Smith, 2008).

Dando respuesta a las cuestiones planteadas, se identifican dos supuestos básicos: la cooperación y la unidad transfronteriza. En primer lugar, muchas regiones europeas han apostado por la cooperación transfronteriza, entendida como un proceso realizado de forma igualitaria entre los actores territoriales de ambos lados de la frontera, como medio para la captación de recursos (Futmair, 2014). En segundo lugar, el marketing transfronterizo se basa en la construcción de una narrativa de unidad transfronteriza, derivada de los aspectos compartidos a ambos lados de la frontera (como la lengua, la identidad, el comercio, la movilidad laboral transfronteriza, el paisaje y las infraestructuras), que se incorpora en la marca territorial. De la misma forma, se procede a la creación y uso de eslóganes transfronterizos tales como "regiones sin fronteras", "amistad sin fronteras", "cultura binacional", entre otros ejemplos similares. Ambas dinámicas suponen una defronterización desde la escala local y regional; es decir, un declive de la significación del territorio nacional y de las fronteras como elementos claves para la organización de la vida social, el desarrollo económico y el orden político.

Sin embargo, el predominio analítico europeo ha imbuido el corpus teórico de una eurocentralidad temática y conceptual. Como se mostrará con el caso analizado, aquellos supuestos no siempre se cumplen cuando se aplican a otros contextos fronterizos. Contrariamente al supuesto cooperativo, las estrategias transfronterizas pueden también realizarse en el marco de una relación desigual e, incluso, con la ausencia de los actores de un lado. En relación al segundo, la construcción de la narrativa transfronteriza y de la marca territorial no se basa únicamente en elementos compartidos (en la búsqueda de la 
unidad); basta con que sean complementarios y, por lo tanto, se pueden basar en las diferencias existentes a ambos lados de la frontera, reforzando las asimetrías. En este caso, estas dinámicas refuerzan la diferenciación fronteriza.

\section{Caso de estudio: la región transfronteriza Tamaulipas-Texas}

La región fronteriza del norte de Tamaulipas (región Frontera) y del sur de Texas (Laredo y Rio Grande Valley) corresponde a la parte baja del río Bravo / Grande, incluido el delta (Mapa 1). En comparación con otras regiones de la frontera México-Estados Unidos, ésta se caracteriza por un elaborado sistema urbano constituido por varias ciudades gemelas. Asimismo, el sur de Texas es el área de Estados Unidos con la más alta proporción de población mexicanoamericana (por encima del $85 \%$ en los condados fronterizos), lo que ha llevado a identificarla como una región culturalmente mexicana (Arreola, 2002) a la vez que la población angloamericana y mexicana anglizada tiene un mayor peso entre las élites (Valerio-Jiménez, 2013). Los ejes de articulación de la región transfronteriza son el río, la frontera (delimitada a partir de aquél) y los puentes internacionales que conectan ambos lados. Los dinamismos urbano, demográfico y económico están liderados por los pares Matamoros-Brownsville, Reynosa-McAllen y Nuevo Laredo-Laredo. Históricamente es posible identificar tres estructuras económicas dominantes: desde su colonización en el siglo XVIII hasta fines del siglo XIX esta región se había mantenido escasamente poblada (especialmente el lado norte) y con un débil desarrollo económico, basado eminentemente en la ganadería; desde inicios del siglo XX, con la construcción del ferrocarril y de infraestructura hídrica, y hasta la década de 1960 la agricultura fue dominante, que supuso una primera etapa de crecimiento demográfico y urbano; y actualmente la estructura económica se basa en la industria, el comercio internacional, la construcción y los servicios, cuyo desarrollo motivó el crecimiento urbano y demográfico. 


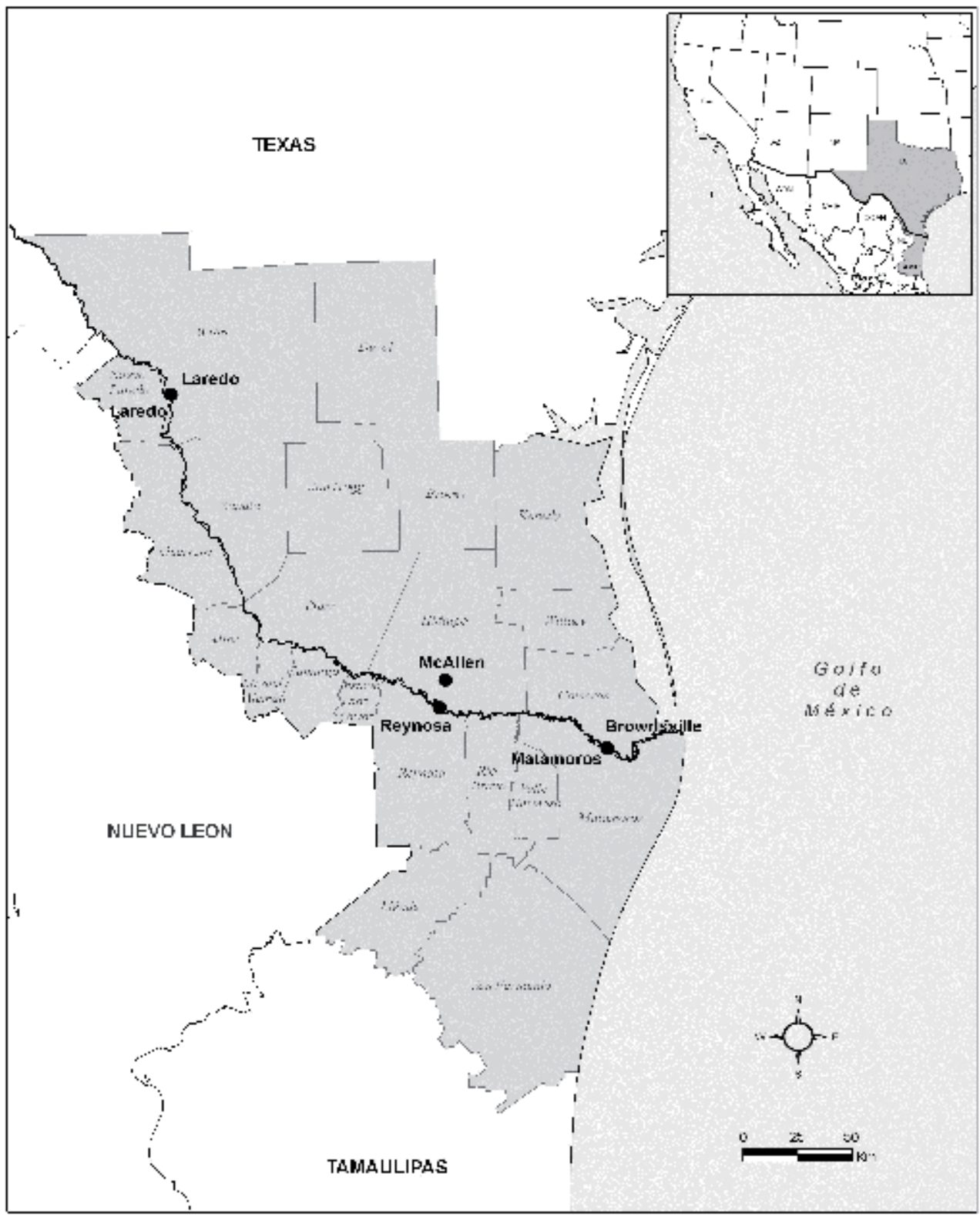

Mapa 1: Área de estudio: la región transfronteriza Tamaulipas-Texas. Fuente: Unidad de Servicios Estadísticos y Geomática (El Colegio de la Frontera Norte). 
Las estrategias de desarrollo regional emprendidas en el lado texano desde mediados del siglo XIX incluyeron el marketing territorial. En el este de Texas esta práctica se inició en la década de 1830 -con la colonización angloamericana- y se extendió y popularizó durante el último tercio del siglo XIX, en un nivel muy superior al resto de Estados Unidos (Rozek, 2003; Jordan, 1980). Aquellas estrategias de marketing, resumidas en la frase "Come to Texas", se dirigieron a la atracción de residentes (ya fueran inmigrantes de Estados Unidos o de Europa) e inversiones, mediante la publicación de guías, almanaques y panfletos. En la región fronteriza de Tamaulipas no se desarrolló un proceso similar sino hasta recientemente, el cual se ha vinculado en gran medida a las estrategias texanas, por su carácter transfronterizo, de las cuales se beneficiaban directa e indirectamente. Sin embargo, esto acarreó una dependencia que, como se mostrará, se ha mantenido en cierto grado hasta la actualidad. Para su exposición, los resultados se presentan en función de las tres grandes estrategias identificadas, dirigidas a la: 1) captación de inversiones y residentes para el desarrollo agrícola y comercial (1880-1950); 2) captación de visitantes (1920-actualidad); y 3) captación de inversiones para desarrollo industrial (1965-actualidad).

\section{Captación de inversiones y residentes para el desarrollo agrícola y comercial (1880-1950)}

Desde mediados del siglo XIX se intentó en ambos lados de la frontera la construcción de grandes sistemas de riego en las márgenes y la llanura deltaica del río Bravo / Grande. Se argumentaba que permitiría el desarrollo de una agricultura comercial y el crecimiento demográfico en una región escasamente poblada, pero de gran importancia geopolítica. Ello no fue posible hasta la llegada del ferrocarril (primero a Laredo y Nuevo Laredo en 1881 y, después, en Brownsville, McAllen, Matamoros y Reynosa a partir de 1903) y otras innovaciones tecnológicas (como el vagón frigorífico), que determinaba la distribución y comercialización de la producción. Con el objetivo de captar inversionistas y residentes se desarrollaron estrategias de marketing territorial, primero en Laredo y, posteriormente, en Rio Grande Valley, mientras que en el norte de Tamaulipas no hay constancia de procesos similares. Las estrategias texanas incluían el "otro lado", aunque solamente como un elemento más de la marca territorial. Esta inclusión se daba a menudo en forma de comparación con México, en un contexto en el que se quería superar a aquél país, lo que constituía una llamada a transformar el lado texano en una región más desarrollada y poblada que el mexicano. 
Las primeras experiencias se dieron en los años 1880 en Laredo por parte de dos organizaciones económicas privadas, la Laredo Immigration Society y la Laredo Improvement Company, la primera centrada en la captación de residentes y la segunda con un carácter mucho más comercial (Adams, 2008; Young, 2004; 1998). Mediante la promoción territorial se acentuaban unas "grandes oportunidades" económicas en el ámbito del transporte y del comercio internacional, dirigidas a la atracción de residentes angloamericanos y nuevas empresas, la expansión de los negocios locales existentes y el fomento de la actividad inmobiliaria y el crecimiento urbano. Se buscaba "to make Laredo the great international commerce center of the southwest" (General Directory of the City of Laredo, 1889 citado en Young, 2004, p. 133) y, por lo tanto, ser la entrada a Estados Unidos y a México.

Las estrategias en Rio Grande Valley se centraron en el desarrollo agrícola y no tanto en el comercial. La primera experiencia, en 1893 para Brownsville, la lideró W.H. Chatfield (en representación de varios inversionistas del norte de Estados Unidos) y contó con el apoyo financiero de distintos comerciantes de Brownsville y, en menor cantidad, de Matamoros. El objetivo era atraer inversionistas y residentes angloamericanos al lado texano, en tanto que la región ofrecía "a grand opportunity for amassing wealth and providing lucrative employment for an immense number of people" (Chatfield [1893] 1991, p. 1). Con un discurso "romántico" (Montejano, 1987), promocionó al "Country of the Lower Rio Grande" como un lugar de tierras fértiles y de clima agradable (para el cultivo, el trabajo y la salud), aunque subdesarrollado por su lejanía geográfica y, con un claro tinte xenófobo, una excesiva influencia mexicana. Por falta de condiciones la campaña no tuvo éxito; sin embargo, con la llegada del ferrocarril y la construcción de los sistemas de irrigación, se articuló una estrategia más ambiciosa. Entre 1905 y 1941 varias empresas agrícolas y desarrolladoras de bienes raíces crearon y difundieron la marca territorial "Magic Valley", que tuvo un éxito considerable (Brannstrom y Neuman, 2009). Rio Grande Valley se promovió como un lugar ideal para vivir y con grandes oportunidades económicas en el ámbito de la agricultura, representado como un lugar de tierras fértiles e, incluso, con cierto exotismo. Por ejemplo, Kerbey (1939) en un reportaje en The National Geographic Magazine lo asemejaba al egipcio delta del Nilo. Como las anteriores, se buscaba captar inversionistas y residentes angloamericanos.

Los elementos constituyentes de la marca fueron comunes en las diferentes campañas de Laredo y Rio Grande Valley. Se pueden destacar los de carácter geográfico y los histórico-culturales. Entre los geográficos se incluyó la localización fronteriza y el "otro lado", en particular las ciudades gemelas (Nuevo Laredo, Reynosa y Matamoros). En el caso de la pareja Brownsville-Matamoros, Chatfield (1893) las calificó, por primera vez 
en la historia de frontera México-Estados Unidos, como "the twin cities of the border", señalando además que la segunda era "the strongest of the Twin Cities" (p. 31). La proximidad a México constituía una doble ventaja: lugar de origen de mano de obra abundante y barata y como destino turístico exótico y de placer. La primera de estas ventajas reaparecerá en las estrategias para el desarrollo industrial, mientras que la segunda se enfatizará en la atracción de visitantes. De nuevo, Chatfield (1893) representaba Matamoros como un lugar exótico ("oriental looking”) y óptimo para el entretenimiento y el placer.

En relación al aspecto histórico-cultural, la creación de la marca se basó en la política de carácter xenófobo de desasociar el sur de Texas de lo mexicano y, de lo contrario, incentivar su angloamericanización. Los actores buscaban una creciente americanización en detrimento de la mexicanidad. Así, se celebraba el pasado -y no el presente- español y mexicano de las ciudades texanas y el carácter bicultural. Con ello se reforzaba el componente angloamericano, aunque simultáneamente permitía distinguir esta región de otras en Estados Unidos. En esta dirección, las estrategias del sur de Texas resultan parecidas a las desarrolladas en el sur de California, aunque con una menor referencia a lo español y mexicano. En California, entre 1870 y 1950, se reelaboró y celebraba el pasado colonial para crear una mitología evocativa y romántica de la región (Culver, 2009; Watts, 2006; Staples, 2004; Mintz, 2000; Padget, 1995). La reinvención se focalizó en la arquitectura, la toponimia, la gastronomía y otros componentes culturales; así, por ejemplo, se creó y se dotó de estilos arquitectónicos particulares, el "Mission Revival” y el "Spanish Colonial Revival", que reinterpretaban la arquitectura de los siglos XVIII y XIX. Si bien la marca territorial resultante se hizo extensiva al suroeste de Estados Unidos, en el sur de Texas, salvo algunas excepciones, lo mexicano aún era "presente", por lo que se tendió más bien a minimizarlo. Esta característica, como se mostrará, se mantiene en las estrategias contemporáneas para la captación de visitantes. Con todo, al unir ambos aspectos se buscaba a la vez un alejamiento y una proximidad a México.

Los canales de promoción se diversificaron en comparación a los usados al inicio del marketing territorial en Texas. Además de guías promocionales -como las publicadas por Tarver (1889) y Chatfield (1991 [1893]) para Laredo y Rio Grande Valley-, se recurrió a otras acciones: la inclusión de reportajes y anuncios publicitarios en los medios de comunicación de Texas y Estados Unidos -como el artículo de Kerbey (1939)- y la organización de misiones comerciales y visitas para posibles residentes e inversionistas. Este último es el caso de Southwestern Land Company (Sharyland), una de las principales empresas desarrolladoras de Rio Grande Valley, que durante los años 1920-1940 vinculó las oportunidades residenciales y económicas con las actividades turísticas en ambos lados de la frontera. Las excursiones incluían la visita a distintas explotaciones agrícolas y a 
algunas poblaciones del área de McAllen, además de Reynosa. Mención aparte merece la organización y celebración de festivales, de los que destacan "Washington Birthday Celebration" en Laredo (desde 1898), "Charro Days Fiesta" en Brownsville (1938) y "Texas Citrus Fiesta" en Mission (1932); los dos primeros se festejaban conjuntamente con sus respectivas ciudades gemelas, Nuevo Laredo y Matamoros, con eventos de bienvenida en medio de los puentes internacionales. Se trataba de promocionar la región, su economía y cultura, y celebrar la frontera y una relación aparentemente amistosa e igualitaria entre las poblaciones de ambos lados y la angloamericana y mexicana (Oliveras, 2015; Young, 1998). Estas fiestas se mantienen hasta la actualidad, habiéndose añadido otras ciudades fronterizas de ambos lados con eventos similares.

\section{Captación de visitantes (1920-actualidad)}

En este periodo de casi 100 años las estrategias del sur de Texas se han dirigido sobre todo a tres grupos de turistas: a la clase media de Estados Unidos, target mayoritario entre 1920 y 1970; a colectivos específicos de Estados Unidos (y, en ocasiones, de Canadá), los Winter texans (población jubilada durante el otoño y el invierno) y los Spring breakers (población estudiantil durante el periodo vacacional de primavera, el Spring break), a partir de los años 1960 y 1980 respectivamente; y los turistas procedentes de México, especialmente de Tamaulipas y Nuevo León, mayoritarios desde los años 1980. Hasta los años 1950 el principal atractivo del sur de Texas fueron las ciudades fronterizas de México, pero a lo largo de la segunda mitad del siglo XX se desarrollaron recursos propios. En la década de 1950 se inició la construcción y promoción del complejo turístico de South Padre Island; en los años 1970-80 se abrieron varios centros comerciales (shopping malls), siendo los primeros "Amigoland" en Brownsville (1974), "La Plaza Mall" en McAllen (1976) y "Mall del Norte" en Laredo (1977); y se popularizaron las actividades en los espacios naturales (observación de aves y caza y pesca deportiva).

Para los dos primeros grupos, los actores de Tamaulipas no desarrollaron una estrategia propia y simplemente esperaban beneficiarse de las emprendidas en el sur de Texas. Esta actitud pasiva no debe extrañar por cuanto que durante el periodo 1920-1960 las ciudades mexicanas fronterizas vivieron un boom turístico debido a la afluencia de visitantes de Texas y, en general, de Estados Unidos (Arreola y Curtis, 1993). Asimismo, esta actitud queda reflejada en una famosa frase, "What makes Brownsville makes Matamoros", atribuida a una autoridad de la ciudad mexicana y publicada en distintos medios 
estadounidenses durante 1950-701. El posicionamiento de los actores de Tamaulipas fue criticado desde ambos lados de la frontera, especialmente cuando el turismo fronterizo mostraba claros signos de agotamiento, tal y como hicieran Bermúdez (1966) y Dillman (1968). Por otra parte, con el predominio del turismo de origen mexicano en el sur de Texas, Tamaulipas ya no pudo beneficiarse de las estrategias texanas, y sólo recientemente se ha procedido a desarrollar estrategias propias, como para el caso del turismo médico.

La captación de visitantes constituyó otro de los objetivos de las estrategias de la primera mitad del siglo XX. La marca "Magic Valley" incluía la promoción turística de Rio Grande Valley y las ciudades fronterizas de Tamaulipas (Brannstrom y Neuman, 2009; Robinson, 2006). La Southwestern Land Company, por ejemplo, promocionaba Reynosa como un lugar de turismo de placer y entretenimiento (restauración, espectáculos de danza y toros, etc.). En la segunda mitad los actores privados de Rio Grande Valley continuaron con la promoción, en la que también participaron por primera vez actores públicos y, del lado mexicano, algunos negocios turísticos de Matamoros y Reynosa, que se anunciaban en los periódicos locales texanos. La McAllen Chamber of Commerce, en la misma línea que la Southwestern Land Company, condujo una estrategia para la promoción turística de McAllen y Reynosa en Texas. Con leves variaciones se sucedieron varias campañas, empezando en 1951 con "Magic Valley and Old Mexico" (Imagen 1a) hasta "McAllen McNífico" en 1982 (Imagen 1b). A lo largo de estas décadas se perpetuó la marca territorial: el lado texano era representado como un lugar moderno y lujoso, mientras que el tamaulipeco -Old Mexico-era un lugar exótico y de placer; juntos, como una sola región, constituían "an entirely different world" y con un "ideal year-round climate". La región fronteriza de Tamaulipas se subordinaba a Rio Grande Valley y era presentada como el "playground of the Rio Grande Border... Land of Manana [sic]". Esta subordinación culminó finalmente con la idea de que no hacía falta cruzar a México para disfrutarlo; así, en la campaña de 1982, Rio Grande Valley se presentaba como el lugar donde se encuentra "the best of two worlds -Texas and Mexico".

1 "Northern Mexico Export Trade Expands", The Brownsville Herald, 16/09/1956, p. 44; "Two Wheels along the Mexican border", The National Geographic Magazine, 139 (5), mayo 1971, 630-631. 

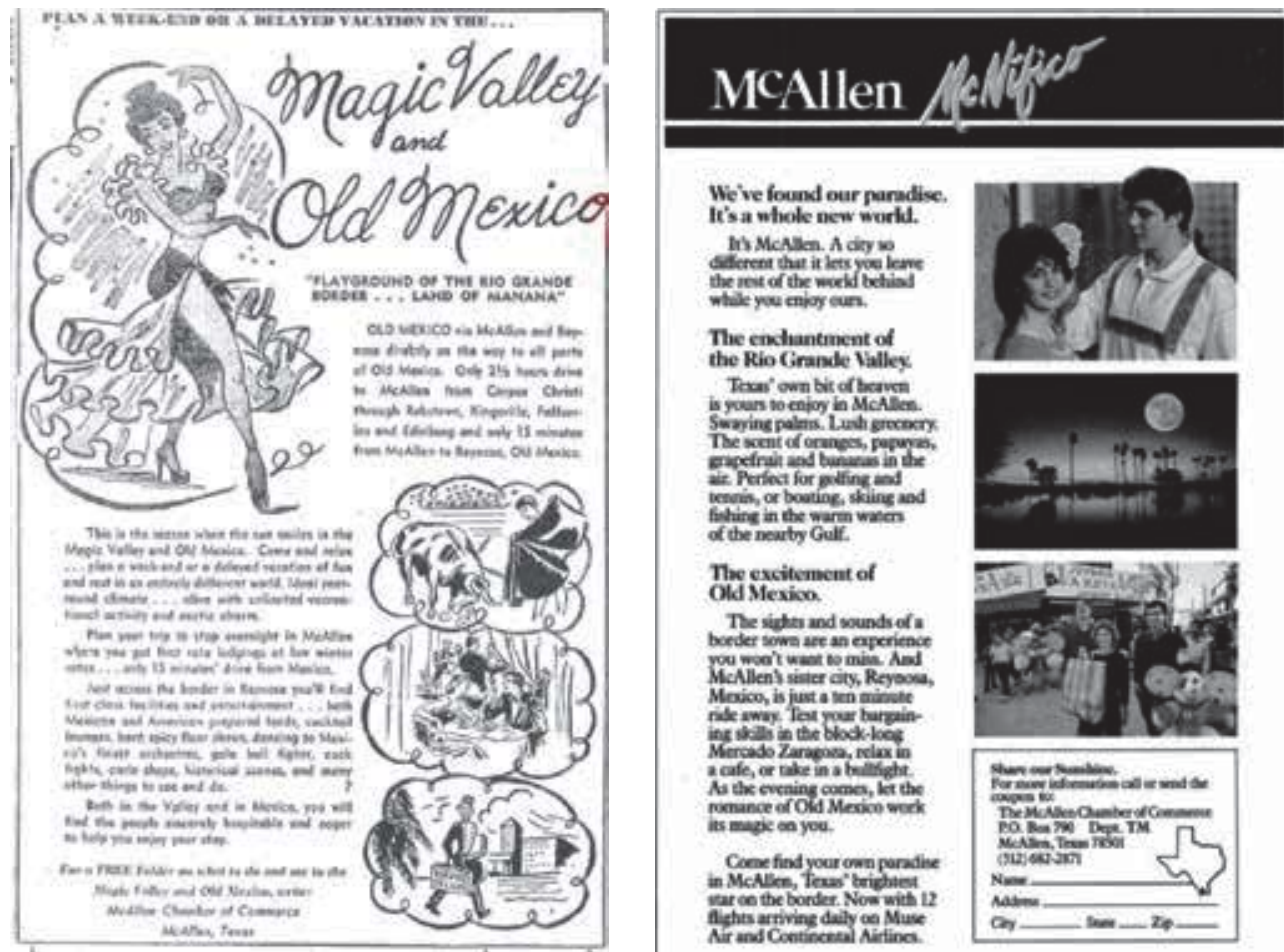

We've found our nanadise It's a whole new world. in McAllan. A dicy wo Silcrent that it lets yos leane the rest of the woeld bechis? while you criog eun.

The enchantanent of the Rio Ginande Valley.

Taxi' own bit of beaven is proars no eniog in McAlles. Swring palas. Luah grocencry

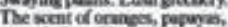
crapefrith and binusts in te.

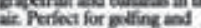
tesuih, or bouting. skings and fishing in the warm wares of the nowby Gulf.

The excitement of Old Mesico.

The sigts and sounds of boeder sowe afe an erperience beeder sowa afe an erpenience MeAllen' sister city, Reyoona, Mexico, is just a len mincite ne vwor. Tat your bargaining wallin the block vac Mercado Zarapoz, relax in Acule, or take in a belight. A the curains comen, ket the ponince of Oxd Mesion werk its magie on you.

Cone find your owa poradine in McAlles, Teas briphter vat ce the bonder. Now with L2 Aights arriving devily os Muse Ar and Continestal Airtines.
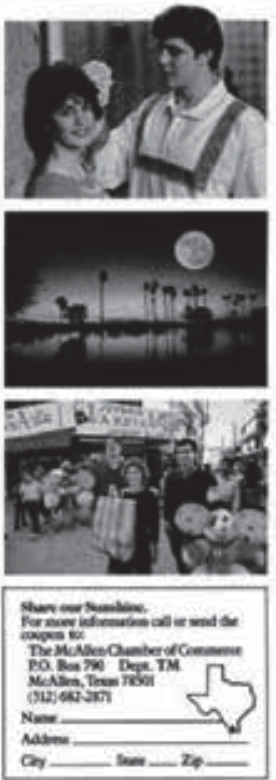

Imagen 1: Magic Valley and Old Mexico (a) y McAllen McNífico (b). Fuente: (a) Corpus Christi Caller-Times, 21/10/1951, p. 45; (b) Texas Monthly, 13 (5) (Mayo 1985), p. 231.

Los actores de Brownsville, por su parte, apostaron por el desarrollo de South Padre Island. La promoción se dirigía a un público general; desde 1982 se apostaría por los Spring breakers, consiguiendo captar unos 80.000-120.000 vacacionistas por año (Laurie, 2008). Por las semejanzas climáticas y paisajísticas, se buscaba asemejar la isla a los destinos turísticos de Florida (Miami y Coral Gables y, específicamente para los Spring breakers, Panama City Beach), a la vez que se reforzaba la marca con la proximidad geográfica a Magic Valley y a México. En esta dirección, la Greater Brownsville Commission creó en 1966 un eslogan para la ciudad, "On the Border, By the Sea"2 (una clara referencia a los atractivos cercanos, México y South Padre Island) y, aunque no obtuvo el impacto esperado, se mantiene hasta la actualidad (Robinson, 2008; Adler, 2000). La marca de South Padre Island combinaba tres elementos: palmeras tropicales, puestas de sol espec-

2 "Brownsville, on the border by the sea, awaits its visitors from the north", The Brownsville Herald, 26/10/1966, p. 13. 
taculares y mujeres bellas, como representación de placer y relajación. La tranquilidad se reforzaba contraponiendo Rio Grande Valley a "the hectic, ulcerous pace" de las áreas metropolitanas de Estados Unidos. México, por su parte, continuó siendo representado como "a world of beauty and tranquility, reeking of lost civilizations and the dead age of Spanish conquest".

Desde los años 1960 la estrategia se dirigió también a la captación y mantenimiento de los Winter Texans, con un énfasis destacado en la salud y los servicios (Simpson y Siguaw, 2013; Dalstrom, 2012; Crompton, Fakeye y Lue, 1992). La marca representaba Rio Grande Valley como un lugar de clima agradable y servicios médicos de calidad, y México, como lugar de artesanías y de servicios médicos y fármacos baratos. La estrategia incluía la organización de distintos eventos dirigidos a este colectivo (festivales de bienvenida y de despedida, ferias de servicios médicos, autocaravanas y de $R V$ parks, etc.), celebrado en las principales localidades de la región. Algunas ciudades gemelas, como Camargo y Rio Grande City, y Nuevo Progreso y Weslaco, celebran eventos conjuntamente (desde 1973 y 1976 respectivamente), emulando los festivales transfronterizos de Laredo-Nuevo Laredo y Brownsville-Matamoros (Oliveras, 2015).

En estas estrategias se movilizaron dos expresiones de uso corriente en Estados Unidos para referirse a México, "Old Mexico” y "Land of Mañana” (o Land of Tomorrow), a menudo con una connotación xenófoba. "Old Mexico" es una expresión afectuosa, asociada a una visión romántica y exótica de México, pero que niega cualquier carácter de modernidad (Arreola y Curtis, 1993). "Land of Mañana”, una metáfora espacial utilizada desde los años 1880, se refiere al lugar donde las cosas se consiguen más lento y más tarde de lo que cabría esperar. Ésta aún se usa para referirse peyorativamente a México (y a América Latina en general) y, a la vez, para representar el suroeste de Estados Unidos como un lugar de vacaciones relajantes y de retiro apacible (Healey y O’Brien, 2007; Zolov, 2004). Sin embargo, en el sur de Texas su significado era ambivalente y adquiría una connotación negativa, más cercana a la representación de México que a la del suroeste. Por esta razón a principios de los años 1970 se optó por dejar de asociar México y/o Rio Grande Valley como "Land of Mañana"3, puesto que, se argumentaba, el "mañana" -es decir, el progreso, la modernidad, el trabajo, la responsabilidad, etc. entendidos según el estándar angloamericano- ya había llegado, aunque hubiera sido con retraso. Con aquel rechazo se perseguía asemejar más el sur de Texas al resto del suroeste y a la vez distinguirla de México, como se pretendía en las estrategias de fines del siglo XIX. El abandono definitivo de las representaciones de "Old Mexico" y "Land of Mañana" se produjo con el

3 "Land of Mañana", The Brownsville Herald, 04/02/1970. 
predominio del turismo mexicano a partir de la década de 1980, dejando de tener sentido la inclusión de México en la marca territorial.

Con un decreciente número de visitantes estadounidenses y un creciente número de los procedentes de México, atraídos principalmente por el turismo de compras, se cambia la estrategia. La McAllen Chamber of Commerce estableció en Monterrey una oficina, Casa McAllen, y publica anualmente desde 1997 la guía turística McNífico, únicamente en español. La localización fronteriza y la proximidad geográfica continúan constituyendo una ventaja competitiva frente a otras ciudades del sur de Texas conocidas por el turismo comercial, como San Antonio y Corpus Christi. Paralelamente, ante la competencia de Laredo y Brownsville, McAllen promociona una imagen urbana chic y sofisticada y de una experiencia más "americana" (es decir, menos "mexicana" que en aquéllas) ${ }^{4}$. La promoción en Tamaulipas y, en general, en el noreste de México de la oferta turística y comercial del sur de Texas también se lleva a cabo mediante publicaciones periódicas creadas específicamente para ello, de las que destacan Texas \& Turismo, publicada en Monterrey desde 1985, y De Shopping en el Valle de Texas, en McAllen desde 2006.

La tendencia a desmexicanizar la imagen territorial del sur de Texas se ha intensificado recientemente, debido a una creciente asociación de México a la inseguridad, violencia, narcotráfico y migración indocumentada, ampliamente difundida en los medios masivos de comunicación. Así, se busca desvincular la marca territorial de la localización fronteriza al haberse convertido ésta última en un elemento negativo de la imagen territorial. Resulta ilustrativo el caso de Brownsville, donde el Brownsville Convention and Visitors intentó reiteradamente (en 2008, 2010 y 2014) sustituir el eslogan de la ciudad ("On the Border, By the Sea") por otro donde México y/o la frontera estén ausentes 5 .

\section{Captación de inversiones industriales (1965-actualidad)}

La industrialización de la frontera de México se impulsó principalmente a partir de tres momentos: el primero, motivado por el Programa de Industrialización Fronterizo

4 "McAllen chamber launches new campaign to attract Mexican shoppers", The Monitor, 22/10/2014, http:// www.themonitor.com/news/local/mcallen-chamber-launches-new-campaign-to-attract-mexican-shoppers/ article_8be52ce0-5992-11e4-823f-001a4bcf6878.html [consultado en: 23/10/2014].

5 "Group hopes to shake off years of shelved slogans", The Brownsville Herald, 15/10/2014, http://www. brownsvilleherald.com/news/local/article_e5c78d70-54e2-11e4-9406-0017a43b2370.html[consultado: $15 / 10 / 2014]$. 
(PIF) de 1965, implementado por el gobierno federal mexicano con el objeto de convertir la franja fronteriza en una zona libre para la localización de industria maquiladora (eminentemente de capital estadounidense), aprovechando el bajo coste laboral; el segundo, la crisis financiera en México de 1988, que hundió el precio del peso en relación al dólar, lo que incentivaba aún más la instalación de empresas estadounidenses; y, el tercero, la entrada en vigor en 1994 del Tratado de Libre Comercio de América del Norte (TLCAN). El proceso de industrialización derivó en nuevas estrategias, en las que participaron actores territoriales de ambos lados. A diferencia de las precedentes, en algunos casos resultaron de la colaboración transfronteriza, aunque los actores del lado texano mantienen el liderazgo y donde los actores de Tamaulipas tienen una participación más o menos activa.

La promoción del PIF para la captación de empresas la realizaron las cámaras de comercio del sur de Texas, que disponían de experiencia en el marketing del lado mexicano, y algunos actores privados de la región fronteriza de Tamaulipas (propietarios de suelo, constructoras y entidades bancarias). En el caso de McAllen y Reynosa, el desarrollo de la estrategia estuvo a cargo de un grupo de iniciativa privada de Reynosa y la McAllen Chamber of Commerce, mientras que el gobierno municipal de Reynosa no mostró un especial interés, más inclinado al desarrollo agrícola (Martin, 1998; Sklair, 1989). La cámara de comercio de McAllen publicó entre 1967 y 1971 varios folletos sobre las oportunidades económicas que ofrecía el PIF y las instalaciones industriales, mercado laboral e infraestructuras de Reynosa. En Matamoros y Brownsville se dio un proceso similar a partir de 1966, fruto de la colaboración de la Brownsville Chamber of Commerce y del Comité de Promoción Industrial de Matamoros (Quintero, 2001; Adler, 2000; Sklair, 1989).

Hasta mediados de los años 1970 se obtuvieron escasos resultados. Las estrategias sufrían de una debilidad: la falta de infraestructura y experiencia industrial, que dificultó tanto la construcción de la marca territorial como la captación de empresas. Se basaron casi exclusivamente en la promoción de las oportunidades económicas y comerciales que ofrecía el PIF, unos costos laborales más bajos en México, y la proximidad geográfica y la localización fronteriza. A pesar de que Tamaulipas no contaba con un sector industrial destacable, las estrategias buscaron asemejar la región fronteriza a los países industrializados de Asia oriental, como Japón y Taiwán. La comparación se sustentaba en ser lugares donde era factible la localización de compañías de capital estadounidense, y frente a los cuales México ofrecía una ventaja competitiva por la proximidad geográfica. Como se argumentó entonces, "the Mexican border is your Japan at the back door" (Brownsville Chamber of Commerce, citado en Sklair, 1989, p. 118). Si bien la estrategia se benefició de la inercia de las estrategias anteriores, no se remitieron a las marcas territoriales que 
habían creado. Se evitó referirse a Tamaulipas como "Old Mexico" y "Land of Mañana", puesto que no representaban un México industrioso ni competitivo. Sin embargo, la marca construida continuaba respondiendo, y reforzaba, la segregación espacial delimitada por la línea fronteriza. En Rio Grande Valley se ubicaba la calidad de vida, los servicios especializados, las oficinas de las empresas, mientras que en la región fronteriza de Tamaulipas, las plantas maquiladoras, la mano de obra barata y, consiguientemente, los impactos ambientales, sociales y en la salud. En este sentido, el argumento dirigido a los empresarios era: "You come to Brownsville, you live in the U.S., you keep your children in U.S. schools, you cross the river to work during the day, you get paid in dollars, you come home to the U.S. at night" (Brownsville Chamber of Commerce, citado en Adler, 2000, p. 258). En esta cita no se expresa directamente la frontera, como reflejo de una aparente unidad transfronteriza; sin embargo, se sustituye por el río, que deviene el límite de la diferenciación espacial de los usos y funciones.

Las estrategias de marketing se retomaron con las ventajas derivadas de la crisis financiera de México y el TLCAN. A lo largo de los 25 años siguientes, la colaboración entre McAllen y Reynosa ha tenido una mayor continuidad y éxito, que se ha traducido en mayores resultados (Pipkin, 2012; Hirschberg, 2011; Martin, 1998). Por el contrario, Brownsville y Matamoros se caracterizan por la discontinuidad y una predominio de la acción aislada, aunque desde ambas se reconoce la dimensión transfronteriza. En el primer caso, después de los esfuerzos de la McAllen Chamber of Commerce, la iniciativa la tomó la McAllen Economic Development Corporation (MEDC), una organización público-privada sin ánimo de lucro creada en 1987. Desde su fundación contempló el crecimiento económico de ambas ciudades de forma inseparable, apostando por la promoción del sector industrial. Desde fines de los años 2000, Reynosa adquirió un rol más activo, culminado por ahora con la creación en 2014 del Consejo Binacional para el Fomento Económico (Oliveras, 2014). En las segundas, por el contrario, la colaboración transfronteriza entre los gobiernos locales y/o los organismos económicos públicoprivados ha sido poco habitual, de corta duración (sujeta a los cambios políticos en los gobiernos locales) y escasos resultados. Sirvan de ejemplo los dos intentos realizados entre 2004 y 2009: la "Alianza del Río, Golfo y Costa", integrada en 2005 por Matamoros, Brownsville y South Padre Island, para la promoción conjunta de las tres ciudades como una región industrial, comercial y turística; y la "Estrategia Binacional para la Promoción de Inversiones Matamoros/Brownsville" que en 2007-2009 reunió los gobiernos de Tamaulipas y Matamoros, el Comité para el Desarrollo Económico de Matamoros (CODEM), el Brownsville Economic Development Council y la Greater Brownsville Incentives Corporation. 
En ambos pares las estrategias se han dirigido a la promoción territorial y la captación de inversores y empresas a nivel internacional mediante la participación en ferias y exposiciones industriales, foros internacionales y encuentros con site selectors; el contacto y asistencia legal a empresas que pudieran localizarse; y la difusión de información económica regional mediante publicidad, reportajes en revistas especializadas (como Site Silection Magazine) y páginas web (como "Brownsville-Matamoros United"6 fruto de la "Estrategia Binacional para la Promoción de Inversiones Matamoros/Brownsville") (Imagen 2a). Asimismo, tanto las estrategias como la colaboración transfronteriza continúan basándose en una distribución de funciones y de usos del suelo definida por las asimetrías entre ambos lados de la frontera; el caso Reynosa-McAllen resulta ejemplar. La MEDC se encarga del diseño y desarrollo de la marca y la promoción territoriales y del contacto con empresas, a la vez que el gobierno de McAllen zonifica y desarrolla áreas de oficinas, residenciales y recreacionales para población de clase media y media-alta (entre la cual el personal directivo y calificado). Por su parte, los actores públicos y privados de Reynosa deben proveer de parques industriales para las plantas maquiladoras e infraestructuras de transporte, con los servicios adecuados (agua, energía, pavimentación, etc.). A pesar de esta división, en los eventos promocionales es habitual la presencia de empresarios y representantes públicos de ambas ciudades.
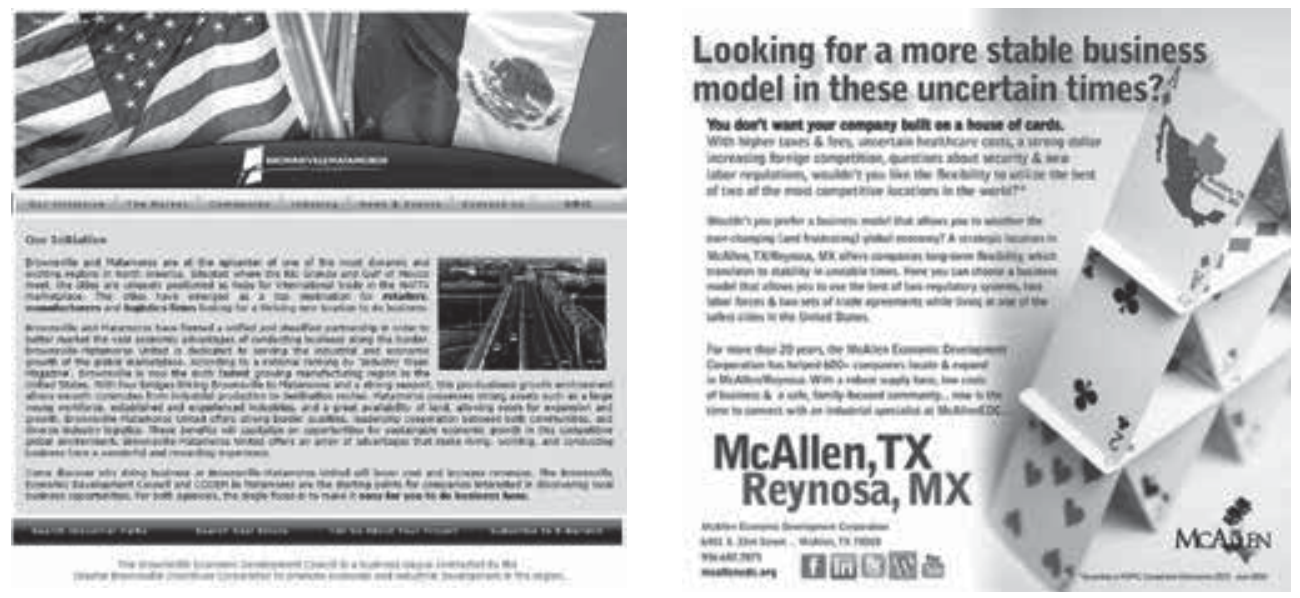

Imagen 2: Brownsville-Matamoros United (a) y "Looking for a more stable business model in these uncertain times?" (b). Fuente: (a) Brownsville Economic Development Council, 2009; (b) Site Selection Magazine, 55 (4), (julio 2010).

6 http://oceanstechnology.com/ [consultado en 02/10/2014]. 
La marca territorial no difiere sustancialmente de la construida en los años 19601970; si acaso, se sofisticó. Se basa en una centralidad sobredimensionada de la industrialización y en una continua expansión como motores del desarrollo regional. Esta sobredimensión ha llevado a sobrevalorar la localización geográfica o el peso dentro del sector industrial; en la campaña "Brownsville-Matamoros United", por ejemplo, se anunciaba que "Brownsville and Matamoros are at the epicenter of one of the most dynamic and exciting regions in North America". Los elementos centrales son la localización fronteriza de las ciudades, la disponibilidad de puentes internacionales y las asimetrías entre ambos lados. Así, la MEDC destaca entre las ventajas competitivas el uso de "the best of two regulatory systems, two labor forces \& two sets of trade agreements", a la vez que los inversores y empresarios pueden trabajar en Reynosa ("a robust supply base, low costs of business") mientras pueden vivir en McAllen ("a safe, family-focused community"). A pesar de la diferenciación espacial, se difunde a la vez la visión romántica de "una sola región": en "Brownsville-Matamoros United" se indicaba específicamente que se trata de " 2 Countries, 1 Region, 1 Culture". A diferencia de la estrategia de los años 1960, en ésta ya era posible enfatizar la expertise industrial y la formación técnica, como hizo la CODEM en la década de 1990 (Zárate, 1999); incluso se ha destacado el escaso poder de los sindicatos obreros, como la MEDC en relación a Reynosa. Por último, ante la reciente percepción de inseguridad en la región fronteriza de México y en Rio Grande Valley, que afecta de manera directa a la economía regional y a la marca territorial, la estrategia se ha tenido que adaptar; McAllen, por ejemplo, se promociona como "one of the safest cities in the United States" (Imagen 2b).

\section{Conclusiones}

Retomando lo que se apuntaba al inicio, las regiones transfronterizas y otros espacios interregionales ofrecen un caso particular para el análisis del marketing territorial. Las funciones de este proceso estratégico (presentar información, incrementar la demanda y diferenciar) para la captación de recursos son desarrolladas de forma conjunta por distintos lugares, en este caso geográficamente contiguos y localizados a ambos lados de una frontera internacional. Como resultado de la eurocentralidad temática y conceptual en los estudios de caso, a menudo se asume que el marketing transfronterizo es fruto de procesos de cooperación y donde la construcción de la marca territorial se basa en los aspectos comunes y/o compartidos a ambos lados de la frontera. El caso aquí analizado muestra que estos dos supuestos teóricos no necesariamente se cumplen siempre. Por el contrario, aunque el lugar objeto de marketing tenga una dimensión transfronteriza, el 
desarrollo de las estrategias puede ser resultado de la acción de los actores territoriales de un sólo lado de la frontera y/o darse una relación de subordinación y dependencia entre los actores de ambos lados; paralelamente, la construcción de la narrativa transfronteriza y, por consiguiente, de la marca territorial puede basarse en las asimetrías territoriales entre ambos lados. Estas dos dinámicas no solo se basan en la diferenciación fronteriza sino que la manifiestan y refuerzan, contrariamente a lo que a menudo se supone.

En relación al primero, la mayor parte de las estrategias desarrollados en la región transfronteriza estudiada han sido iniciativa y lideradas por los actores del sur de Texas. Esto se puede ver muy especialmente en aquéllas dirigidas a la captación de residentes, inversores comerciales y agrícolas, y visitantes. Ello se debe a que el lugar objeto de desarrollo regional es el sur de Texas, no la región transfronteriza. En esas estrategias los actores territoriales incluyeron Tamaulipas / México como un elemento más de la marca territorial del sur de Texas, motivados por la vecindad fronteriza y la proximidad geográfica. Los actores de Tamaulipas, por otra parte, no desarrollaron una estrategia propia, sino que mantuvieron una actitud pasiva y esperaban beneficiarse de la texana, muy particularmente en la captación de turistas. En la captación de inversiones industriales se detecta de forma más clara el recurso a procesos cooperativos, aunque limitados y sujetos a distintos obstáculos. En este caso, el lugar objeto de desarrollo industrial es la región fronteriza de Tamaulipas, aunque en el diseño e implementación del marketing ha predominado la acción de los actores del sur de Texas y la dependencia de Tamaulipas. A pesar de esta relación desigual, como se desprende de las estrategias más recientes, desde el lado mexicano se está procediendo a buscar un rol más activo y un proceso más colaborativo. Resta para otra ocasión el análisis de sus limitaciones, obstáculos y éxitos.

En segundo lugar, esta relación desigual se manifiesta también en la marca territorial construida, cuya base se apoya en las asimetrías territoriales. Es especialmente evidente en las estrategias de desarrollo agrícola e industrial, donde Tamaulipas constituye una fuente de mano de obra abundante y barata, y para la localización de las actividades y usos del suelo de menor valor añadido (incluida la externalización de los impactos ambientales, sociales y en la salud), mientras que en Texas se localizan las actividades y usos de mayor valor, y la población de un mayor nivel socioeconómico. Asimismo, por medio de los elementos que integran la marca se sobrevalora el sur de Texas a la vez que se busca la desmexicanización y/o angloamericanización de dicha región, sobre todo cuando la mexicanidad se representa como negativa en la imagen territorial; simultáneamente se incluye una representación subordinada de lo mexicano para el norte de Tamaulipas. 
Además de la frontera espacial y sociopolítica, por último, en las estrategias analizadas también confluye la frontera cultural. Así, se puede argumentar que tanto la participación y relación entre los actores territoriales como la construcción de la marca territorial forman parte del propio proceso de avance del espacio sociocultural angloamericano sobre el mexicano. Cabe recordar que este último no se restringe únicamente al espacio estatal mexicano sino que se extiende a ambos lados de la frontera política. Se detecta una continuidad desde el siglo XIX en la representación por parte de los actores anglotexanos de una superioridad ante lo mexicano, tanto en la dimensión cultural como la espacial. Sin embargo, la representación espacial del lado texano resulta ambivalente, puesto que a menudo la representación del lado mexicano construida en Estados Unidos también se aplica al sur de Texas, "demasiado" mexicano para el estándar (xenófobo) angloamericano. Ejemplo de ello es el devenir de la marca territorial de "Land of Mañana" (aplicada peyorativamente a México y positivamente al suroeste de Estados Unidos): ante la incapacidad de los actores del sur de Texas de trasladar este significado positivo a su región, desecharon su reproducción para referirse a Tamaulipas. Más recientemente se puede observar en la aplicación para Rio Grande Valley de la imagen negativa derivada de la inseguridad y el narcotráfico, por lo que sus actores buscan distanciarse de México como forma de negación de dicha imagen.

\section{Referencias}

Adams, J. A. (2008). Conflict and commerce on the Rio Grande: Laredo, 1755-1955. Texas, Estados Unidos: Texas A \& M University Press.

Adler, W.M. (2000). Mollie's Job: a story of life and work on the global assembly line. New York, Estados Unidos: Scribner.

Andersson, M. (2007). Region branding: the case of the Baltic Sea Region. Place Branding and Public Diplomacy, 3 (2), 120-130.

-_- . (2010). Place branding and place promotion efforts in the Baltic Sea Region. A situation analysis. Copenhagen, Dinamarca: Baltic Development Forum / Baltic Metropoles.

Arreola, D. D. (2002). Tejano South Texas. A Mexican American Cultural Province. Austin, Estados Unidos: Univeristy of Texas Press. 
Arreola, D. D. y Curtis, J. R. (1993). The mexican border cities. Landscape anatomy and place personality. Tucson, Estados Unidos: The University of Arizona Press.

Bermúdez, A. J. (1966). El rescate del mercado fronterizo: una obra al servicio de México. México, D. F., México: Eufesa.

Brannstrom, C. y Neuman, M. (2009). Inventing the "Magic Valley" of South Texas, 19051941. The Geographical Review, 91 (2), 123-145.

Chatfield, W. H. (1991). The Twin cities of the border and the country of the lower Rio Grande. Brownsville, Estados Unidos: The Brownsville Historical Association [Original: (1893), New Orleans: E.P. Brandao]

Crompton, J. L., Fakeye, P. C. y Lue, Ch. Ch. (1992). Positioning: the example of the lower Rio Grande Valley in the winter long stay destination market. Journal of Travel Research, 31 (2), 20-26.

Culver, L. (2009). Promoting the Pacific Borderlands: leisure and labor in Southern Californias, 1870-1950. En A. McCrossen (ed.), Land of necessity. Consumer culture in the United-States-Mexico borderlands (pp. 168-195). Durham: Duke University Press.

Dalstrom, M. D. (2012). Winter texans and the re-creation of the american medical experience in Mexico. Medical Anthropology: Cross-Cultural Studies in Health and Illness, 31 (2), 162-177.

Dillman, C. D. (1968). The functions of Brownsville, Texas and Matamoros, Tamaulipas: twin cities of the Lower Rio Grande. Tesis de doctorado. The University of Michigan.

Futmair, S. (2014). Opportunities and challenges of cross-border place branding. The case of Euregio. Tesis de maestría. University of Twente / Westfälische Wilhelms Universität Münster.

Healey, J. F. y O’Brien, E. (eds.) (2007). Race, ethnicity, and gender: selected readings. Thousand Oaks: Sage.

Hirschberg, V. A. (2011). Economic development and planning in Reynosa since 1990. Ongoing Studies in Rio Grande Valley History, 10, 301-358. 
Hospers, G. J. (2004). Place marketing in Europe. The branding of the Oresund Region. Intereconomics. Review of European Economic Policy, 39 (5), 271-279.

- _ _ - (2006). Borders, bridges and branding: the transformation of the Oresund Region into an imagined space. European Planning Studies, 14 (8), 1015-1033.

Ioannides, D., Nielsen, P. Å. P. y Billing, P. (2006). Transboundary collaboration in tourism: the case of the Bothnian Arc. Tourism Geographies, 8 (2), 122-142.

Jordan, T. G. (1980). Immigration to Texas. Boston: American Press.

Kerbey, M. (1939). The Texas Delta of an American Nile. The National Geographic Magazine, 75 (1), 51-97.

Lamour, C. (2014). Territorial reputation beyond state borders? Metropolitan images in European borderlands. Place Branding and Public Diplomacy, 10, 19-31.

Laurie, J. (2008). Spring break: the economic, socio-cultural and public governance impacts of college students on Spring Break Host Locations. Tesis de doctorado. University of New Orleans.

Martin, P. (1998). On the frontier of globalization: development and discourse along the Rio Grande. Geoforum, 29 (3), 217-235.

Mintz, S. (ed.). (2000). Mexican american voices. New York, Estados Unidos: Brandywine Press.

Montejano, D. (1987). Anglos and mexicans in the making of Texas, 1836-1986. Austin: Texas University Press.

Nilsson, J. H., Eskilsson, L. y Ek, R. (2010). Creating cross-border destinations: INTERREG programmes and regionalisation in the Baltic Sea area. Scandinavian Journal of Hospitality and Tourism, 10 (2), 153-172.

Oliveira, E. (2014). Cross-border Place Branding. Zooming in the potential case of Galicia and Northern Portugal. Comunicación presentada en Conference on Interregional Place Branding, Lübeck, Alemania. 
Oliveras, X. (2014). La acción transfronteriza de los gobiernos locales en un contexto de endurecimiento fronterizo y crisis económica. El caso de la región Tamaulipas-Texas. Carta Económica Regional, 113, 13-35.

- - — . (2015). Fiestas transfronterizas y representaciones espaciales en la frontera México-Texas. Economía, Sociedad y Territorio [en prensa].

Padget, M. (1995). Travel, exoticism, and the writing of region: Charles Fletcher Lummis and the "Creation" of the Southwest. Journal of the Southwest, 37 (3), 421-449.

Pasquinelli, C. (2013). Competition, cooperation and co-opetition: unfolding the process of inter-territorial branding. Urban Research and Practice, 6 (1), 1-18.

Pipkin, S. D. (2012). Repertoires of development in economic divergence on the U.S.-Mexico border. Tesis de doctorado. Massachusetts Institute of Technology.

Prokkola, E. K. (2007). Cross-border regionalization and tourism development at the Swedish-Finnish Border: Destination Arctic Circle. Scandinavian Journal of Hospitality and Tourism, 7 (2), 120-138.

Quintero, C. (2001). La maquila en Matamoros: cambios y continuidades. En M. E. de la O y C. Quintero (Coords.), Globalización, trabajo y maquilas: las nuevas y viejas fronteras en México (pp. 73-110). México, D. F., México: Plaza y Valdés.

Robinson, R. (2006). Promoting Valley Tourism in the 1920s: a case of competition and ambivalence. Further Studies in Brownsville and Matamoros History, 7, 209-219.

- _- _. (2008). Why Brownsville Failed to Become “The Next Coral Gables”. Journal of South Texas, 21 (2), 188- 211.

Rozek, B. J. (2003). Come to Texas. Enticing Immigrants, 1865-1915. College Station, Estados Unidos: Texas A\&M University Press.

Šerić, N. y Vitner, S. (2011). Brand management in the practice of cross-border tourist destinations. Academica Turistica, 4 (2), 89-99.

Simpson, P. M. y Siguaw, J. A. (2013). Lifestyle and satisfaction of winter migrants. Tourism Management Perspectives, 5, 18-23. 
Sklair, L. (1989). Assembling for development: the maquila industry in Mexico and the United States. New York, Estados Unidos: Routledge.

Smith, P. J. (2008). Branding Cascadia: considering Cascadia's conflicting conceptualizations. Who gets to decide? Canadian Political Science Review, 2 (2), 57-83.

Staples, J. P. (2004). Constructing the Land of Sunshine: Charles Fletcher Lummis and the marketing of a Post-Frontier West. Tesis de doctorado. The University of Arizona.

St. John, R. (2009). Selling the border: trading land, attracting tourists, and marketing american consumption on the Baja California Border, 1900-1934. En A. McCrossen (ed.), Land of necessity. Consumer culture in the United-States-Mexico borderlands (pp. 113142). Durham, Estados Unidos: Duke University Press.

Tarver, E. R. (1889). Laredo, the gateway between the United States and Mexico: an illustrated description of the future city of the great Southwest. Laredo, Estados Unidos: Daily Times Print / Laredo Immigration Society.

Valerio-Jiménez, O.S. (2013). River of hope. Forging identity and nation in the Rio Grande borderlands. Durham: Duke University Press.

Watts, J. A. (2006). Photography in the Land of Sunshine: Charles Fletcher Lummis and the Regional Ideal. Southern California Quarterly, 87 (4), 339-376.

Witte, J. J. (2014). Best practices of cross-border place branding in Europe. Comunicación presentada en Conference on Interregional Place Branding, Lübeck, Alemania.

Young, E. (1998). Red men, princess Pocahontas, and George Washington: harmonizing race relations in Laredo at the turn of the century. The Western Historical Quarterly, 29 (1), 48-85.

- - _- (2004). Catarino Garza's Revolution on the Texas-Mexico Border. Durham: Duke University Press.

Zárate, A. (1999). Huevos de oro. El desarrollo industrial de Matamoros. Matamoros, México: Instituto Tecnológico de Matamoros.

Zolov, E. (2004). Showcasing the "Land of tomorrow": Mexico and the 1968 Olympics. The Americas, 61 (2), 159-188. 